\title{
The Chinese Phoenix
}

\section{Anna Perry, Colorado State University, USA}

Keywords: Innovative cotton design, sculptural fashion

Contextual review and concept. Apparel is a carrier of culture (Liang, 2013). The purposes of the design were to 1) combine Eastern culture into apparel design, and 2) demonstrate an innovative way to use $100 \%$ cotton fabric. The inspiration was a supernatural bird, the Chinese phoenix, which is a symbol of purity.
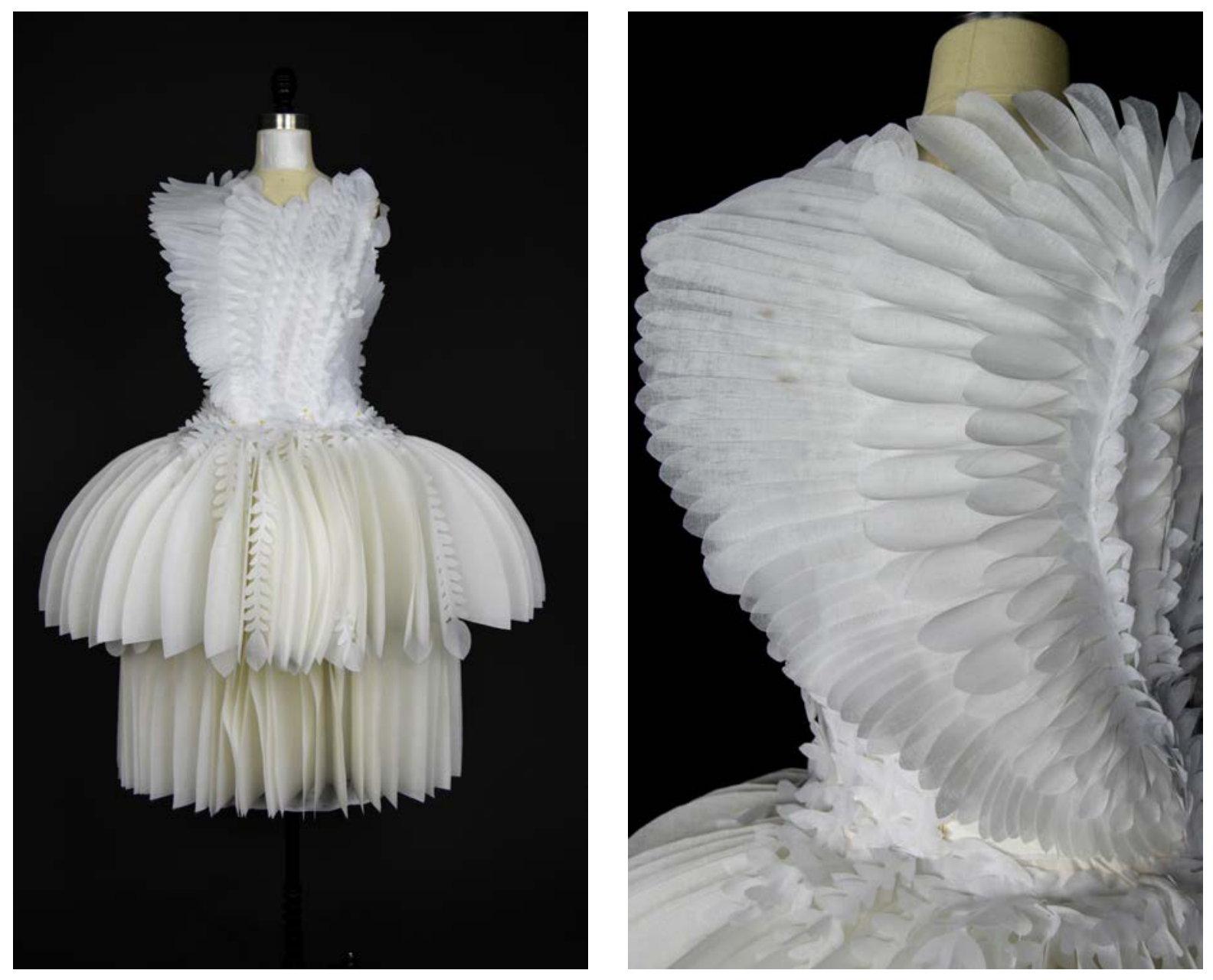

Process, technique, and execution. Design process includes problem identification and prototype development (Parsons \& Campbell, 2004). To solve possible problems, the designer drew 
sketches, made samples, chose white as a design tone for purity, and selected two types of cotton fabric: a soft one for next to the skin, and a stiff one for fabric manipulation and shape maintainability. To develop a prototype, the designer draped the cotton fabric, sewed pattern pieces, and created a rich texture and a visually interesting garment surface. Techniques included draping, paper sculpture method, and fabric manipulation.

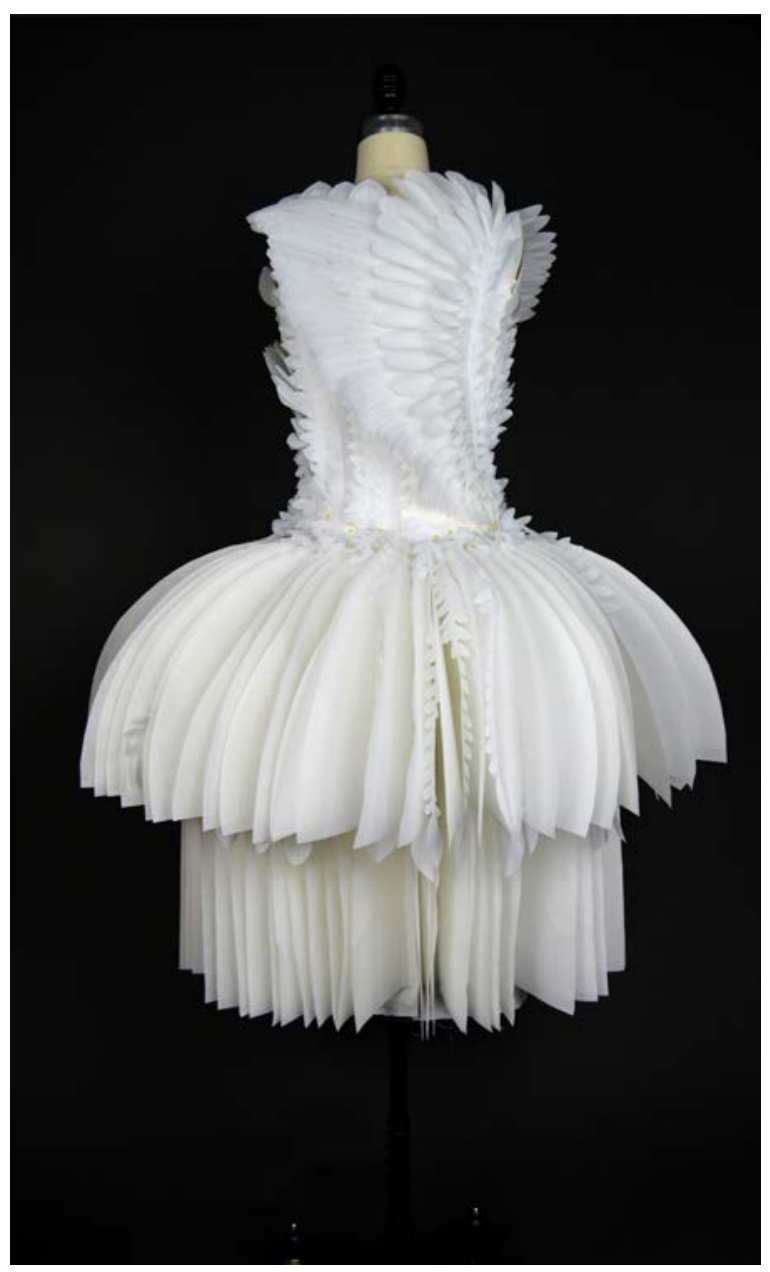

Aesthetics and visual impact. Design principles were applied. Rhythm was created by repeating different sizes of feathers on the top of the garment. Rhythm was also created by repeating same size 2D cotton pieces, creating a 3D skirt with a full 360 degree view.

Cohesiveness. The design process continued to go on until the design met the following standards: 1) realized the purposes, and 2) achieve an overall harmony with aesthetics effect. Eventually, the concept, process, aesthetics, and the final design formed an integrated whole.

Design contribution and innovation. This design refines what is known: the designer applied a paper sculpture method on fabric and created a unique sculptural fashion. This design also contributes to the apparel design field by showing an innovative way to use cotton fabric. Apparel design professionals and students may learn this way and apply it on their own designs.

References

Liang, Y. (2013). Study on clothing marketing strategies based on consuming behaviors of white-collar females. In Du, W (Ed.), Informatics and management science VI (pp. 265272). London: Springer.

Parsons, J. L., \& Campbell, J. R. (2004). Digital apparel design process: Placing a new technology into a framework for the creative design process. Clothing and Textiles Research Journal, 22(1/2), 88-98. 